\title{
RFID Model and Study its Performances
}

\author{
Tirthankar Datta, Ph.D. \\ HOD \& Professor \\ Department of ECE \\ MCKVIE, Howrah, India
}

\author{
Aritra De \\ Assistant Professor \\ Department of ECE \\ CIT, Kolkata, India
}

\author{
Aniruddha Bhattacharjee \\ Student, Final Year \\ Department of ECE \\ MCKVIE, Howrah, India
}

\begin{abstract}
Radio Frequency Identification (RFID) is a generic wireless system which transmits unique identity code using radio waves. This rapidly emerging technology, used to identify objects attached with tags, consists of a reader, tag and wireless channel. The reader \& the tag both consist of a microchip and an antenna performs handshaking whenever the both interact. In the present work, a matlab based RFID system is developed incorporating all the relevant ideas. Performance of the system so developed is also verified with some tag code. Performance of the whole system can be evaluated by changing the coding method, parameters of building blocks, and operation distance. Some simulation results are presented in this paper. A Chaotic Noise block is also incorporated in the system in order to realize actual environmental noise conditions as far as possible. Finally, a detailed study on the Bit-Error-Rate (BER) observed under different conditions is carried out using simulated RFID system.
\end{abstract}

\section{Keywords}

RFID, MATLAB, Reader, Active tags, Passive tags, repeating sequence, backscatter, microchip.

\section{INTRODUCTION}

The concept of RFID explored in 1948 by H. Stockman [1] is first realized as passive radio transponder with memory by Cardullo et al [2]. To investigate the applicability the technology, Los Alamos National Laboratory developed an RFID model to track hazardous nuclear material automatically in early 1970s [3]. Radio-frequency identification (RFID) is an automatic identification method, relying on storing and remotely retrieving data using devices called RFID tags or transponders [4-7]. Since then RFID becomes the natural choice of automatic identification \& traceability processes and enhances its applicability in enormous manner [8-11].

RFID is grouped under the broad category of automatic identification technologies. RFID, the non-contact technology that identifies objects attached with tags, is the next generation wireless communication technology applicable to various areas such as distribution, circulation, transportation, and tracking etc. where Reader and Tags can communicate in a wireless way [12-14]. RFID can also identify mobile objects of high speed. And it can identify a certain amount of Tags simultaneously by its anti-collision mechanism $[15,16]$.

Radio frequency identification technology has become popular as an effective, low-cost solution for tagging and wireless identification. It is obviously a flexible technology and is convenient, easy to use, and most suitable for automatic operation. RFID, essentially, does not require contact or line of site to operate. However, it can function under a variety of environmental conditions and ensures a high level of data integrity [17].Tags may be again classified as active, passive or semi passive depending upon the availability of power resources tagged with them. Each tag has distinct advantages and limitations.
Indefinite lifetime, smaller, lighter, small memory size of 32128 bits and less expensive RFID Passive Tags can operate without an on board power source and cover shorter range than active tags. They require high power reader and tags are unable to establish communication among them. However, Active tags eliminate all the shortcomings of their passive counterpart viz. range, communication etc. but are short lived and more expensive compared to passive tags.

Objects on a self or a truck or in a production line can be automatically tracked at anytime from anywhere using RFID system. Thus the primary benefit of RFID is its increased visibility. Moreover, tagged objects can identify themselves; foundation of smart environment provides a major impact in a wide verity of applications. RFID tags will establish communication with its reader, it will detect item instantaneously and relay it to a network. Top notch retail shops [18] employed this technology to smooth functioning of their operation.

RFID systems use different frequencies ranging from low to microwave range. Low frequencies like $30-500 \mathrm{KHz}$ for short-range applications (usually less than two meters), Ultra High frequencies like $860-960 \mathrm{MHz}$, and Microwave frequencies like $2.4-2.5 \mathrm{GHz}$ for longer transmission range (usually more than 27 meters) are mainly used for RFID based application. Various simulation models on RFID viz. simulation platform of RFID operating in UHF region; realization \& optimization of simulated UHF RFID; behavioral modeling and simulation of RFID antennas; realization \& simulation of the hardware for RFID system and its performance study etc. are studied and developed to save the search time for the technologists involved in the fabrication of devices or designing a system [19].

The Ultra High Frequency $(860-960 \mathrm{MHz})$ mode used by the RFID system achieves higher communication ranges, higher data rates and smaller antenna sizes. Hence our work deals with the study of UHF Passive tags of the RFID system. Here we try to develop a RFID system using matlab and study its performance in the frequency range of $860-960 \mathrm{MHz}$.

\section{SIMULATION OF RFID SYSTEM}

In this work, a simulated version of the RFID system is presented. Reader transmits \& receives signals to establish communication with Tags where as Tags sends unique serial id which is decoded by the reader (Fig. 1). 


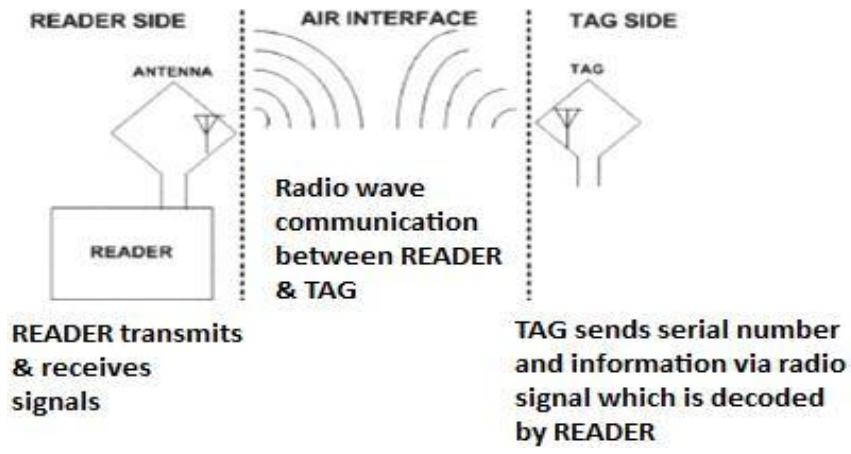

Fig. 1: RFID System

The Forward Link and return link of the system which comprises Reader as well as Tag and the data processor are simulated using MATLAB and Simulink. The present work is inspired by [20] and some additional blocks viz. chaotic noise block etc. are incorporated in order to achieve actual environmental conditions as much as possible. The proposed model is mentioned herewith.

\subsection{Transmission Model}

Tag code is generated by using a repeating sequence block. The code is then modulated with a continuous carrier signal $(A m p=1 \mathrm{~V}$, freq $=900 \mathrm{MHz})$. The modulated tag code is used to enable the tag and the continuous carrier signal is used for backscatter of tag ID during return link. DSB AM is used as the modulation scheme. The modulated signal is amplified, band-limited between $860 \mathrm{MHz}$ to $960 \mathrm{MHz}$ using a Fourth order Band pass Bessel's Filter and transmitted through the antenna. SSB-AM is derived by removing one of the sidebands of DSB-AM and occupies one half bandwidth of DSB-AM. The signal power of SSB is half of the DSB modulation. The noise power in the bandwidth is also half of DSB. Therefore the SNR of SSB and DSB are the same.

\subsection{Model for Channel}

The channel is the wireless media between Reader and the Tag. When the tag enabling modulated signal is transmitted, channel noise is incorporated into the signal and consequently degrades the signal. A numerous factors in channel may be incorporated like-Gaussian noise, Multi path Fading etc. Additive Gaussian noise is simulated by a random generation block. Noise power may be varied by changing the variance of the random number block. As the noise is additive so it is added with the signal by a Summing block. The signal power also attenuates during its transmission from reader to tag and vice-versa.

\subsection{Model of Passive Tag}

Tag has to first identify the code transmitted by the reader and match the code with its own code stored in memory. If matching occurs, Tag will be enabled and backscatter the continuous carrier modulated with Tag ID by changing the reflection coefficient of the tag antenna. The code detection is done by either peak detector or a demodulator with locally generated carrier. To reshape the signal to form the Code in Bit pattern we use 'Gain', 'Saturation', 'Wrap to zero', 'Boolean converter' and 'Logical operator Not' Blocks. Saturation block saturates the signal to an upper and lower threshold values (here upper value $=0.5$ and lower value $=$ 0.4). The Wrap to zero block checks the signal threshold value and forms a pattern which is inverted pattern of the Code. So the actual code is obtained using a 'Not' operation after Boolean converter converts the signal. For Comparison a 'Queue' is used block with push, pop signals and Input and output bit lines. When the start bit is sensed the clock signal starts to push the code bits of the received signal. After all the 10 bits being pushed the clock gives pop signal. At the same time the Tag code is generated by the tag. This is done to synchronize the start bit of receiver code and tag code. The comparison is done by co-relating the two codes using a ' $\mathrm{X}$ NOR' operator. If 10 bits totally matches then the Code word Time segment output will be ' 1 '. To obtain this output a signal block is used. The running sum taken over the code word time period will be 10 , if the tag code and reader code matches, provided sum is taken at the bit rate of the code (which is known). A running sum block and a converter are used for this. For running sum value greater than 9.5 (the switching threshold value) a 3 input switch enables the tag ID transmitter part. Switching from input 3 to input 1 occurs if signal in input 2 crosses the threshold (9.5). Input 1 is the signal transmitted by the reader in Return Link i.e. the continuous carrier, input 2 is the running sum signal and input 3 is connected to ground. If the Tag Code matches, the continuous carrier signal modulated with the Tag ID is reflected back to the receiver by changing the reflection coefficient of the Tag antenna. The simulation is done using a PN sequence generator (generates Tag ID), product and Gain blocks.

\subsection{Receiver model}

The reader receives the direct signal from the transmitter and the reflected one from the surrounding environment. However strength of the signal reflected from the surrounding environment is weak and consequently can be neglected. It is essential to have a high isolation between the transmitting and receiving antenna of the reader. The receiver circuit should have a large dynamic range to handle the relatively large direct signal and detect the weak backscattered signal from the tag.

\section{SIMULATION MODEL}

RFID simulation model is realized utilizing all the relevant considerations. MATLAB is used to develop the same. RFID Reader is simulated in MATLAB environment depicted in Fig. 2 and RFID TAG in Fig. 3. Scopes are employed at different stages to retrieve the relevant waveforms. 


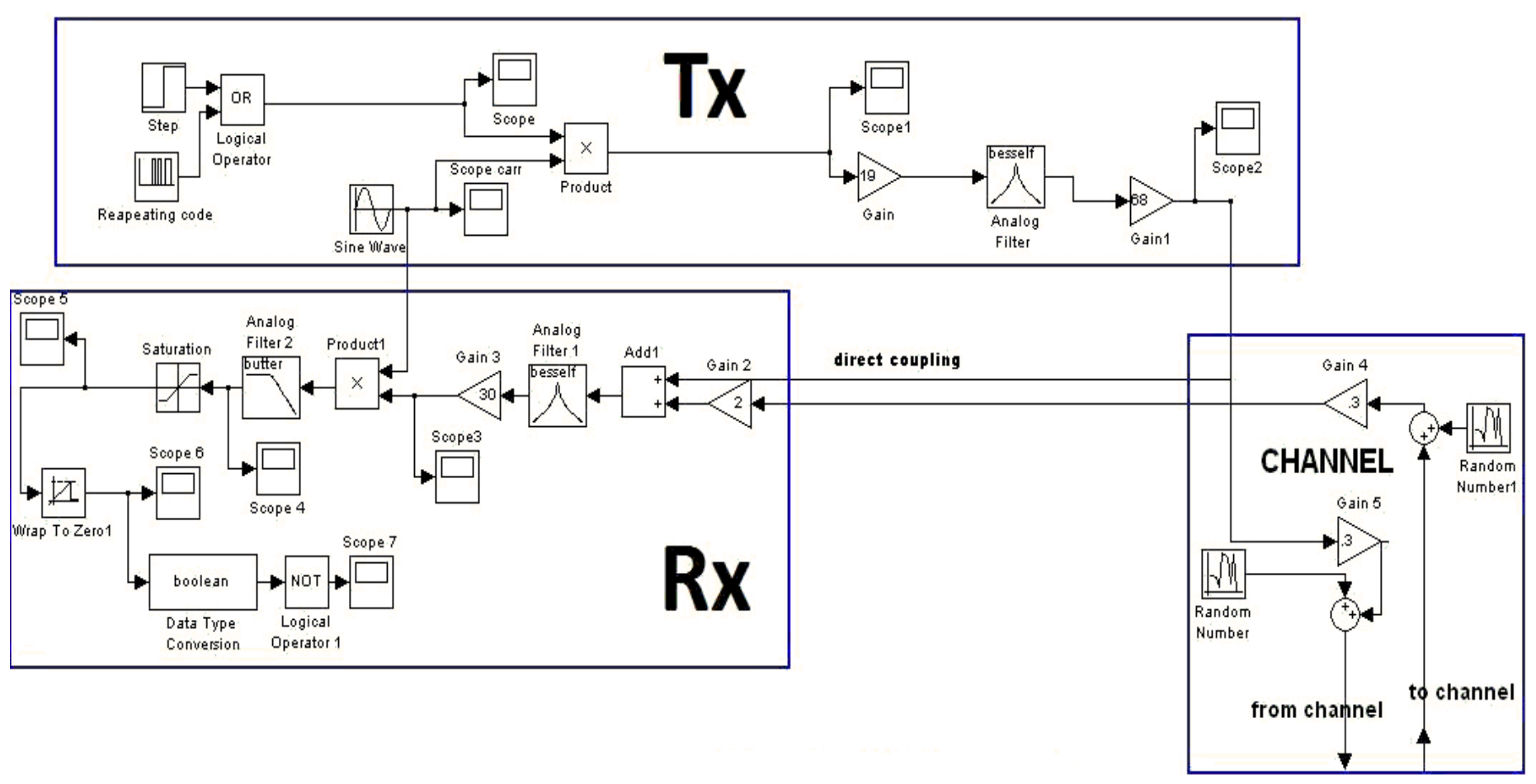

Fig 2: RFID Reader \& Forward Link in MATLAB Simulation Environment

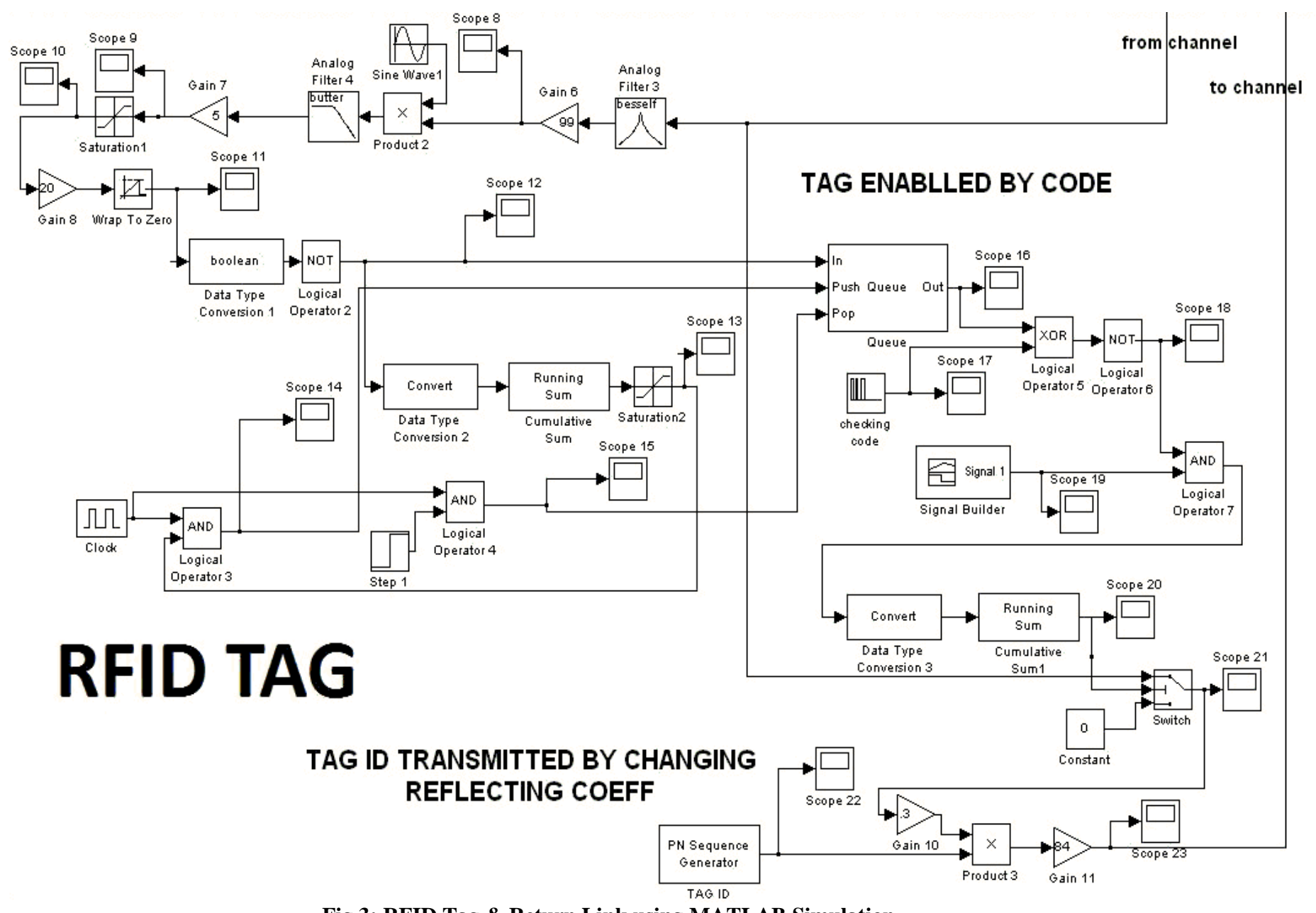

Fig 3: RFID Tag \& Return Link using MATLAB Simulation 


\section{RESULTS AND DISCUSSIONS}

In the present work, an RFID system is in simulated version incorporating all practical environmental aspects. MATLAB and Simulink are employed for system design and performance analysis. All the scope results from both Reader $\&$ Tag are depicted herewith from Fig 4 to Fig. 21 .

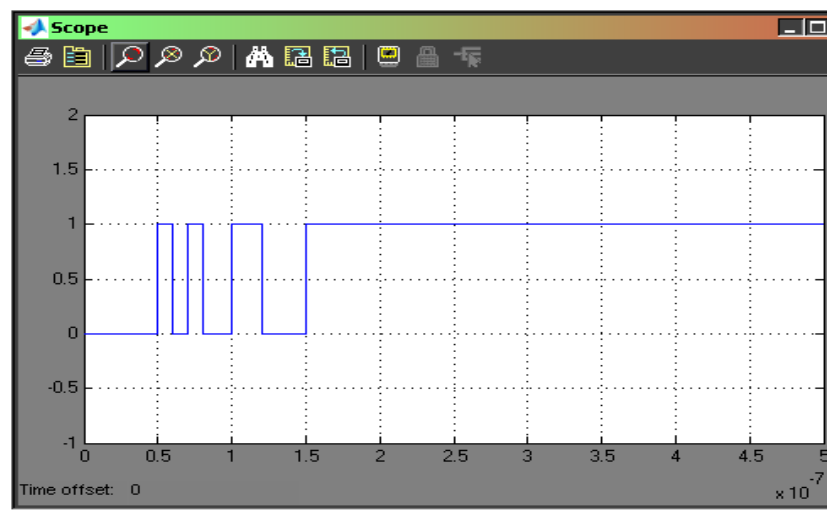

Fig. 4 [Scope]: Tag Code (1010011) appended with a step function is generated

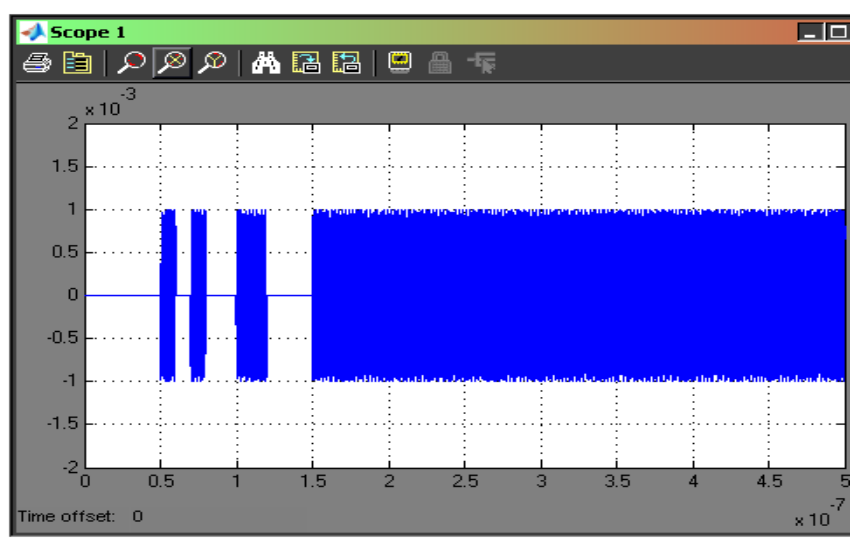

Fig. 5 [SCOPE 1]: The pattern is modulated by DSB AM scheme

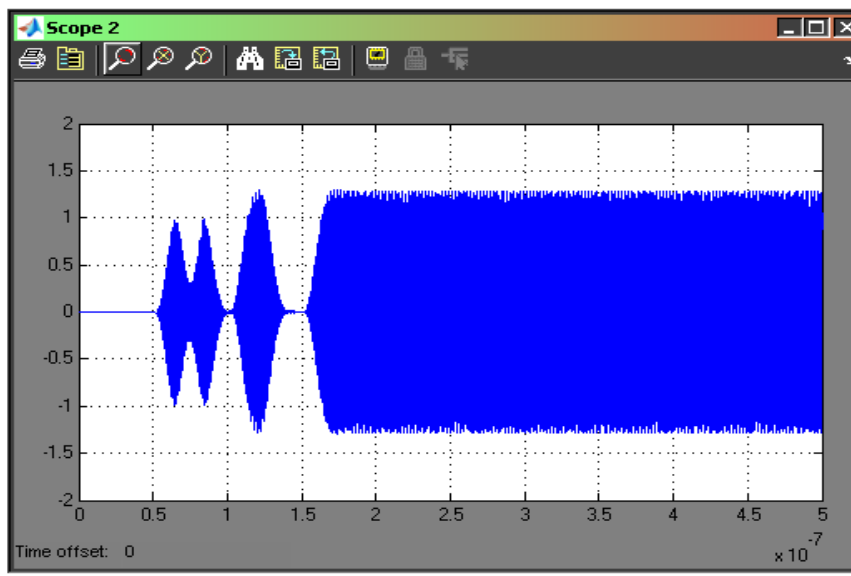

Fig. 6 [SCOPE 2]: Band restricted modulated signal at the output of Analog Band pass filter transmitted by Reader antenna

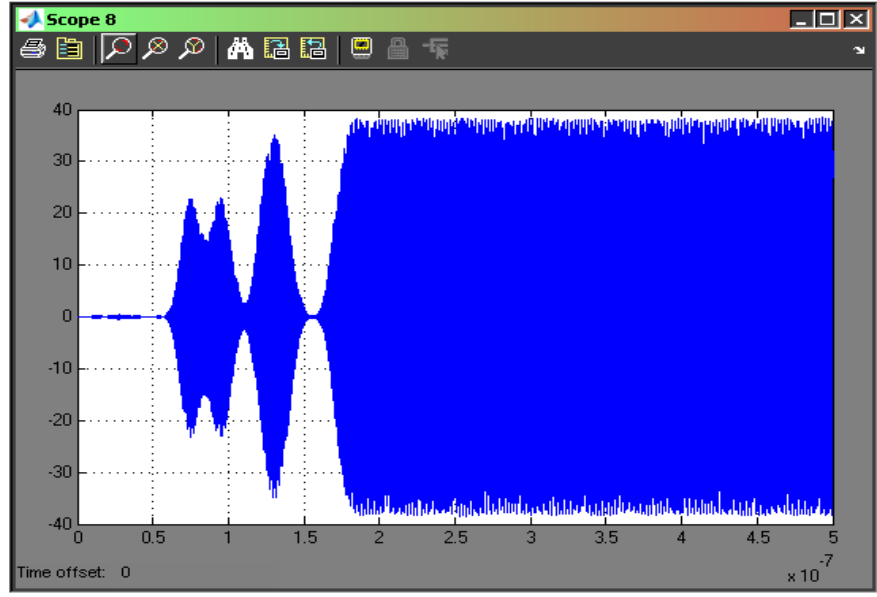

Fig. 7 [SCOPE 8]: Received signal in Tag antenna

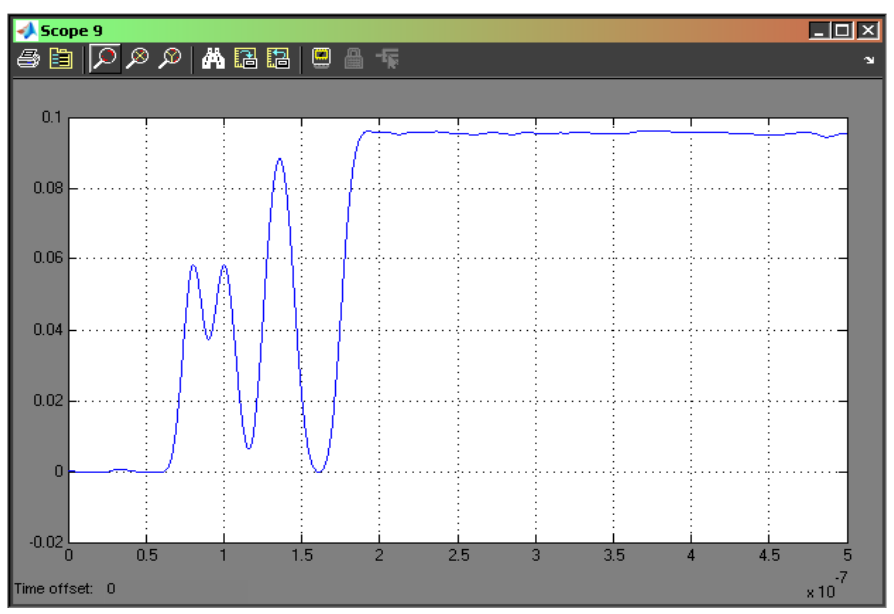

Fig. 8 [SCOPE 9]: Detected signal at the Tag

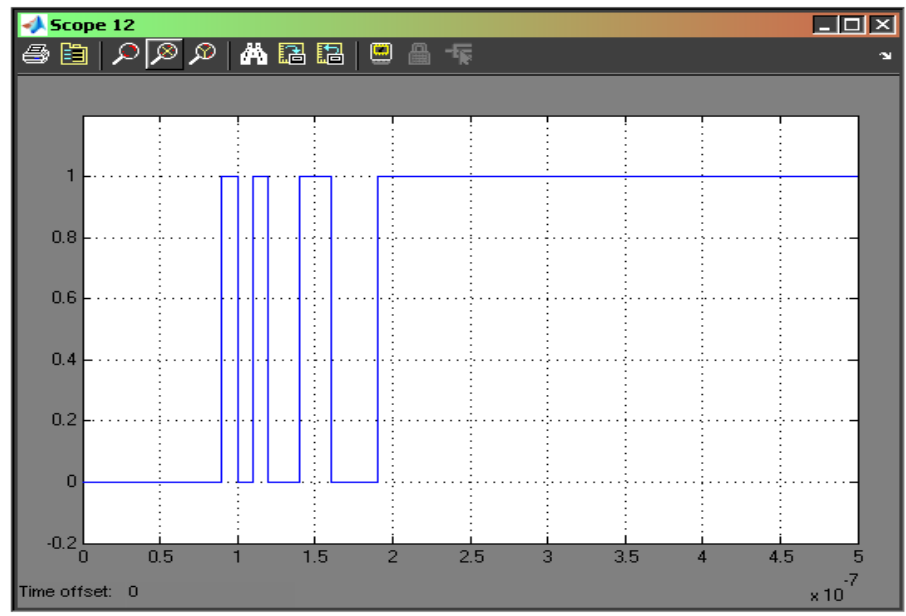

Fig. 9 [SCOPE 12]: Reconstructed Signal with conversion in Boolean form. Code transmitted by Reader 1010011 is found 


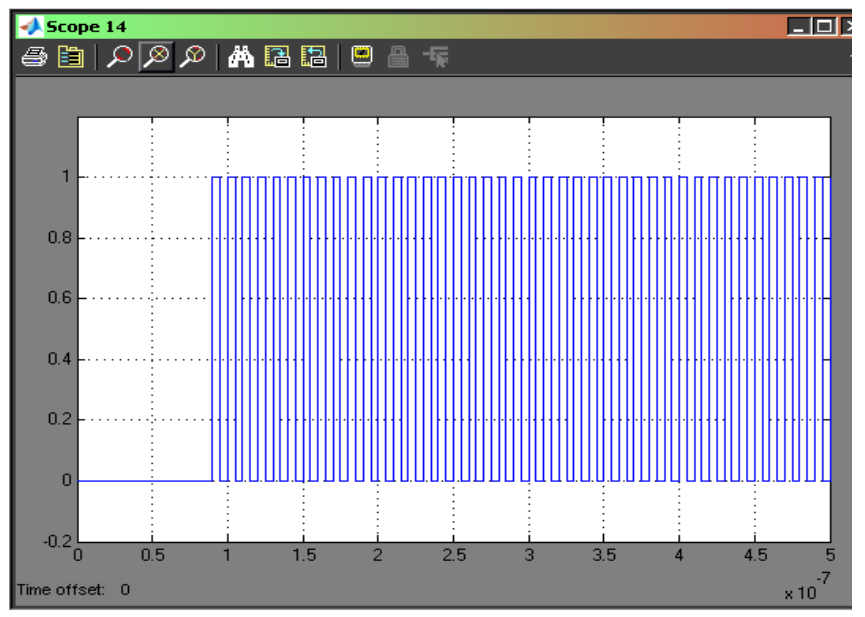

Fig. 10 [SCOPE 14]: Clock signal generated for Push Operation in Queue

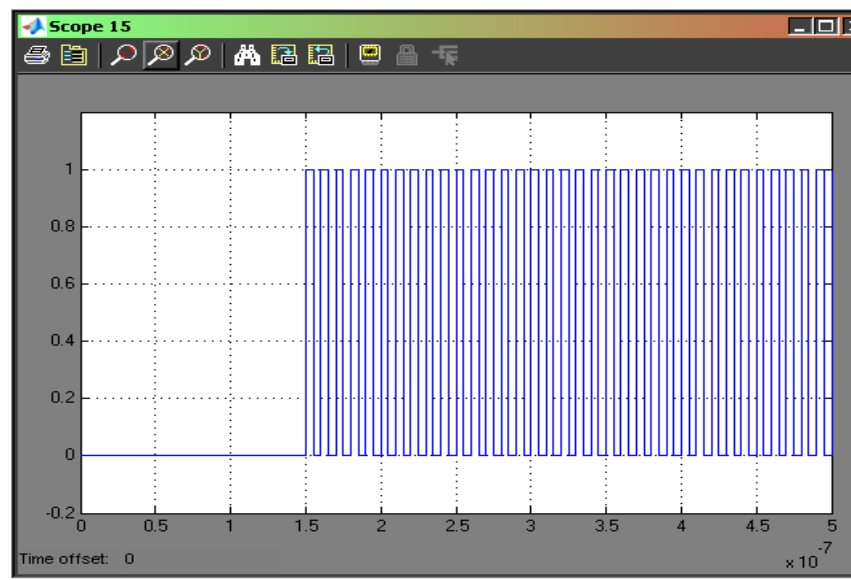

Fig. 11[SCOPE 15]: Clock signal generated for Pop Operation from Queue

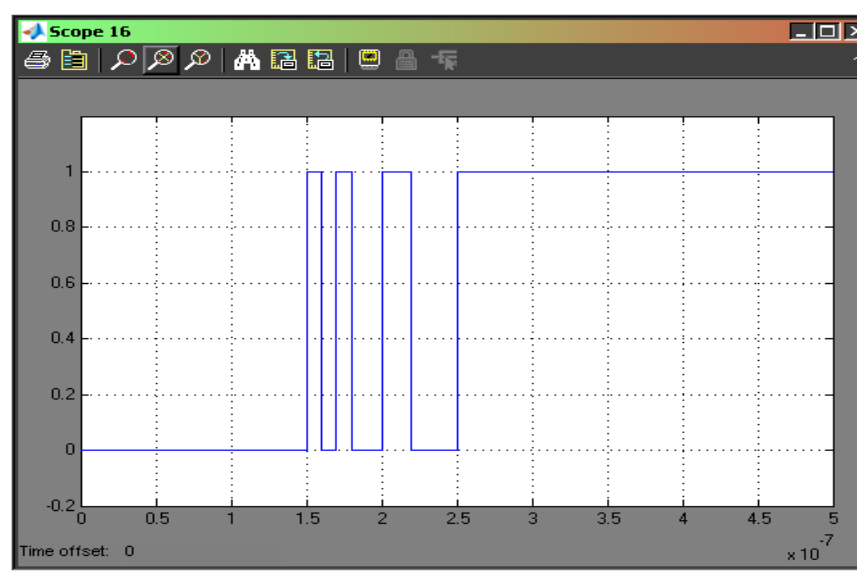

Fig. 12 [SCOPE 16]: The Popped bit pattern from Queue

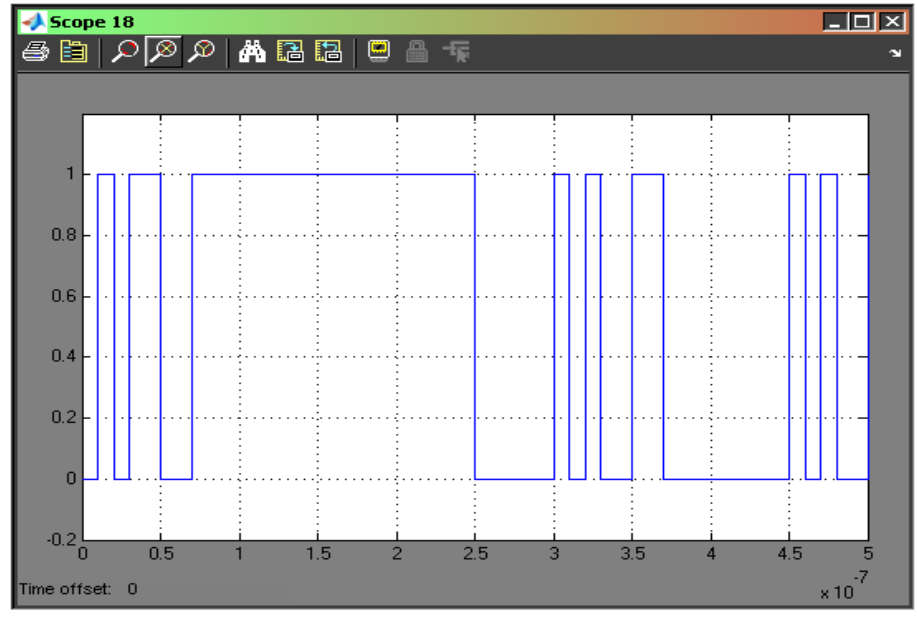

Fig. 13 [SCOPE 18]: The Compared output after Correlating two codes, Tag code and the Code received from Reader

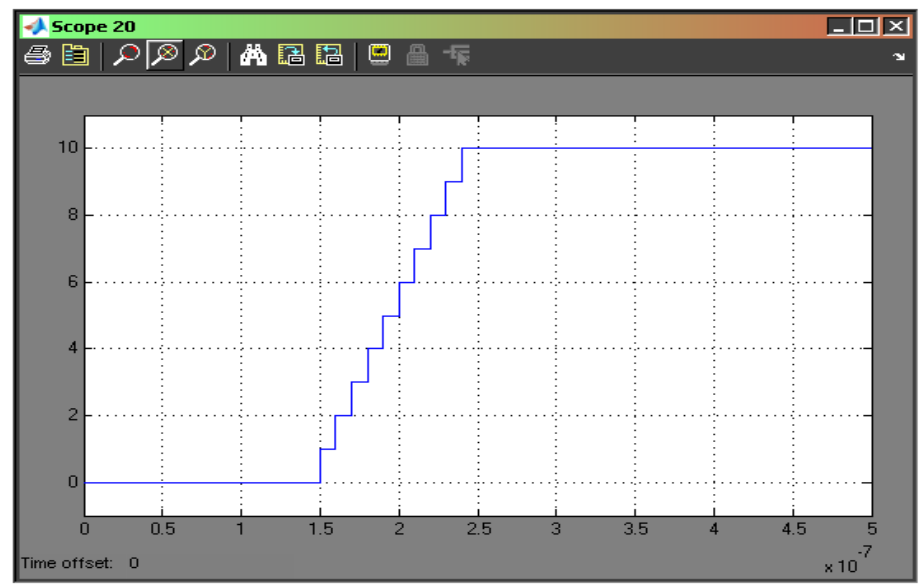

Fig. 14 [SCOPE 20]: The value of Running Sum is 10 since all the code bits matches

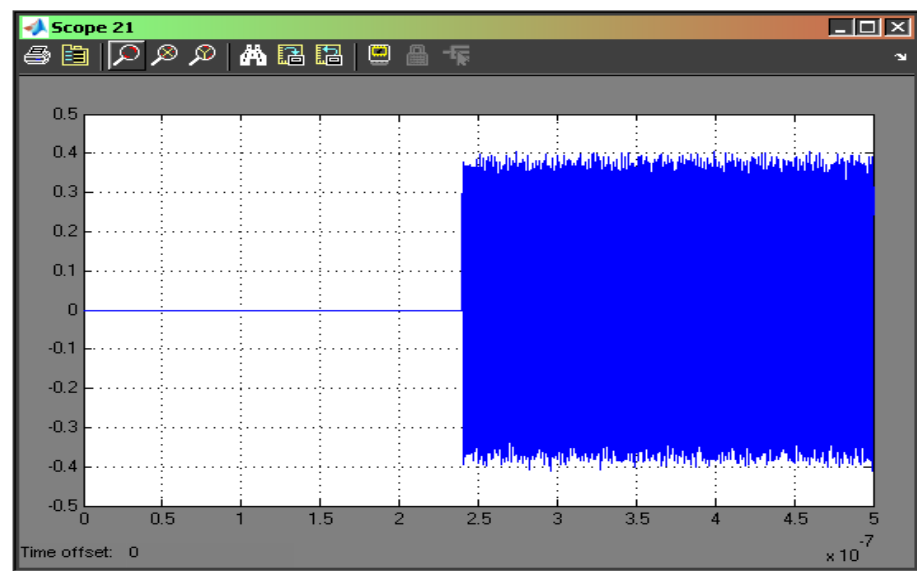

Fig. 15 [SCOPE 21]: After Tag enabling the continuous carrier is allowed by the switch, so that Tag can Power up and transmit the Tag ID 


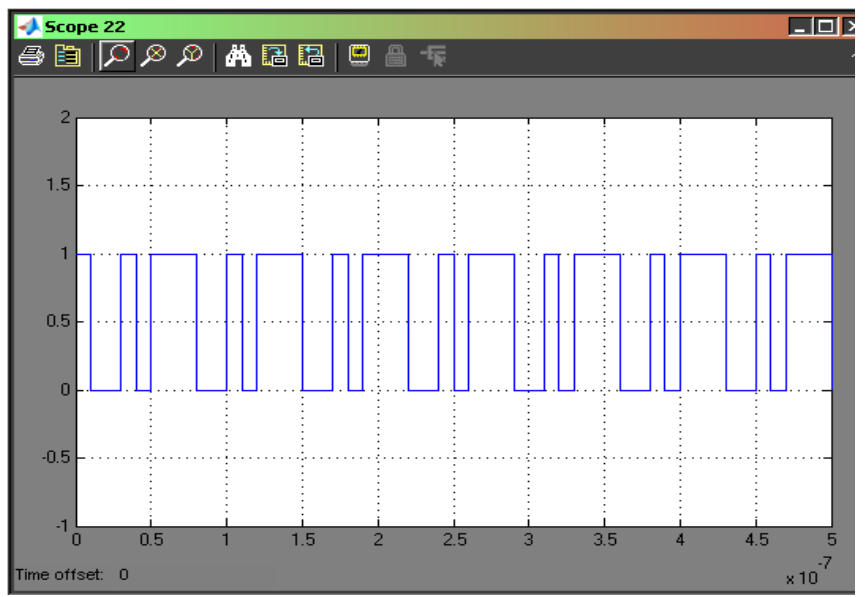

Fig. 16 [SCOPE 22]: Output of PN sequence Generator Simulating Repetitive Tag ID production

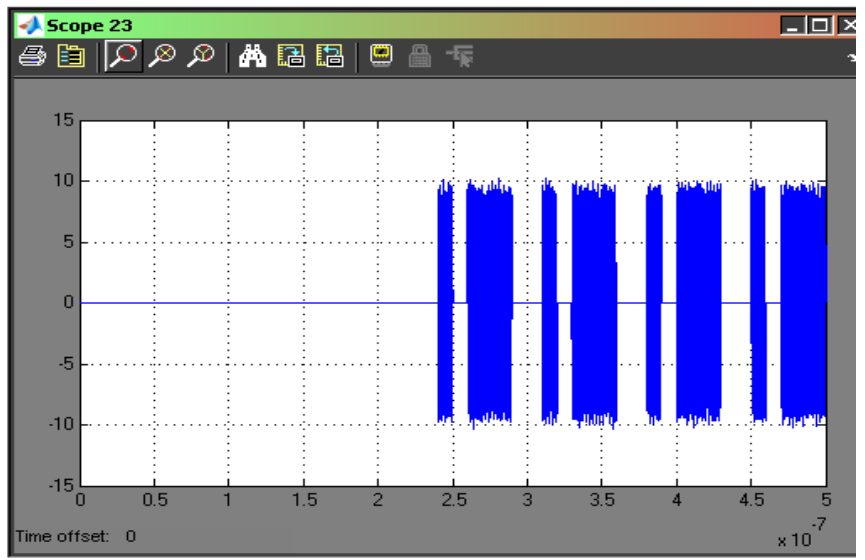

Fig. 17 [SCOPE 23]: The Tag ID modulated by back scattering of Continuous Carrier

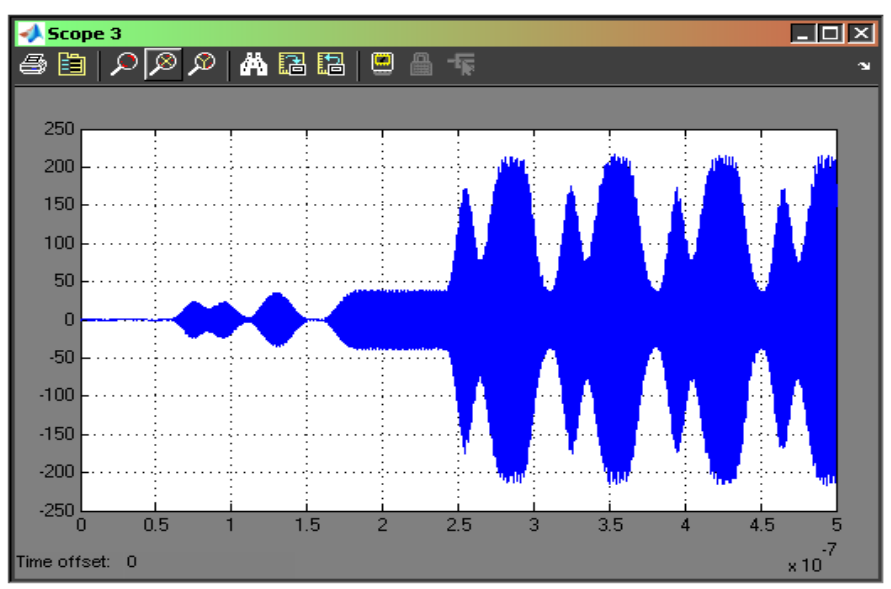

Fig. 18 [SCOPE 3]: Received Tag ID along with the direct coupling effect at the Reader

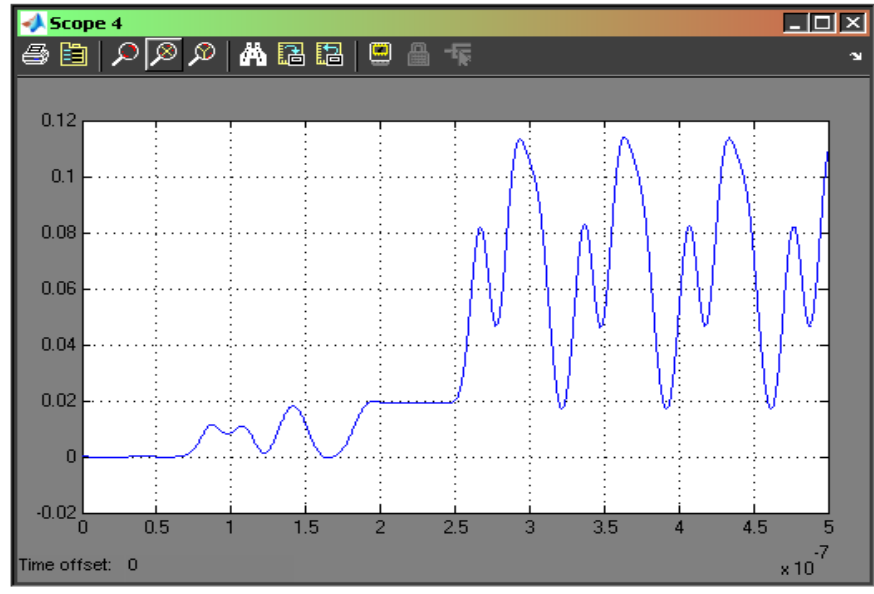

Fig. 19 [SCOPE 4]: The demodulated waveform at the reader $(\mathrm{O} / \mathrm{P}$ of low pass filter $)$

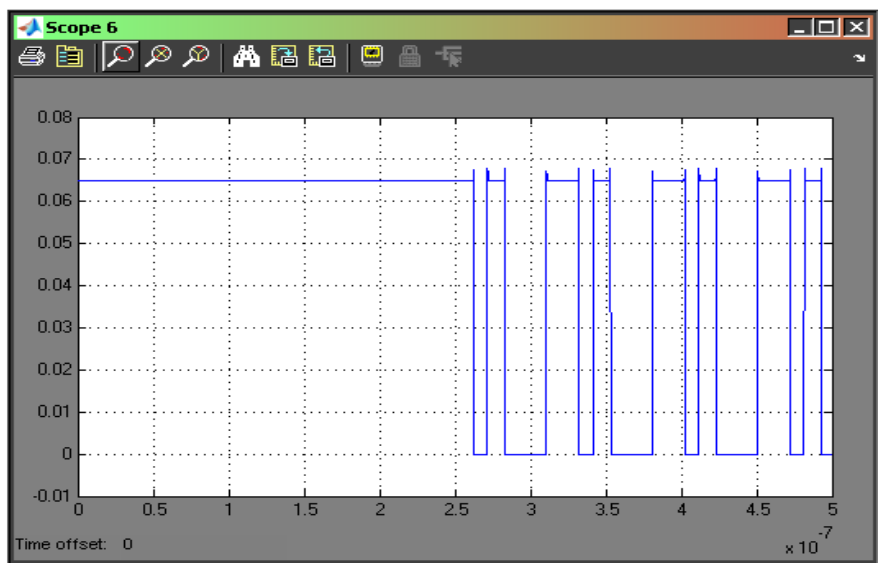

Fig. 20 [SCOPE 6]: Output of Wrap to Zero block for signal conditioning

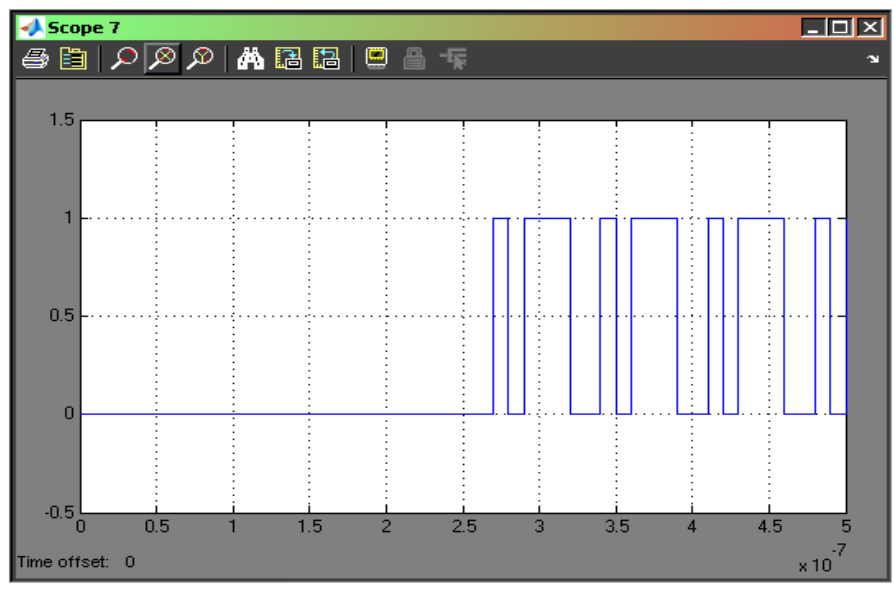

Fig. 21 [SCOPE 7]: The Tag ID reconstructed with conversion in Boolean form. The Tag ID 10111 is obtained at the reader 
Again, the model is also used to calculate the BER at bitrates from $1 \mathrm{Mbps}$ (Mega bits per second) to $20 \mathrm{Mbps}$ at a carrier frequency of $870 \mathrm{MHz}$ and distance between reader and tag = $5 \mathrm{~cm}$. No BER is observed for bitrates below 2Mbps. As bitrate is increased beyond this value, BER also increased and attained a maximum at $20 \mathrm{Mbps}$. However, these values hold good for the simulated model and may exhibit variations in the practical field. It gives us a qualitative idea about the increase in BER with increase in bit-rate. Table.1 gives the data obtained indicating the degradation of identification of higher data-rates. Fig. 22 represents the graphical representation of the data presented in the Table.1

Table.1: Bit rate versus BER

\begin{tabular}{|c|c|}
\hline Bit-rate (Mbps) & BER \\
\hline 10 & 0 \\
\hline 20 & 0 \\
\hline 25 & .081 \\
\hline 28 & .072 \\
\hline 33.3 & .059 \\
\hline 36 & .052 \\
\hline 40 & .1 \\
\hline 60 & .136 \\
\hline 100 & .1 \\
\hline 133 & .15 \\
\hline 150 & .228 \\
\hline 200 & .5 \\
\hline
\end{tabular}

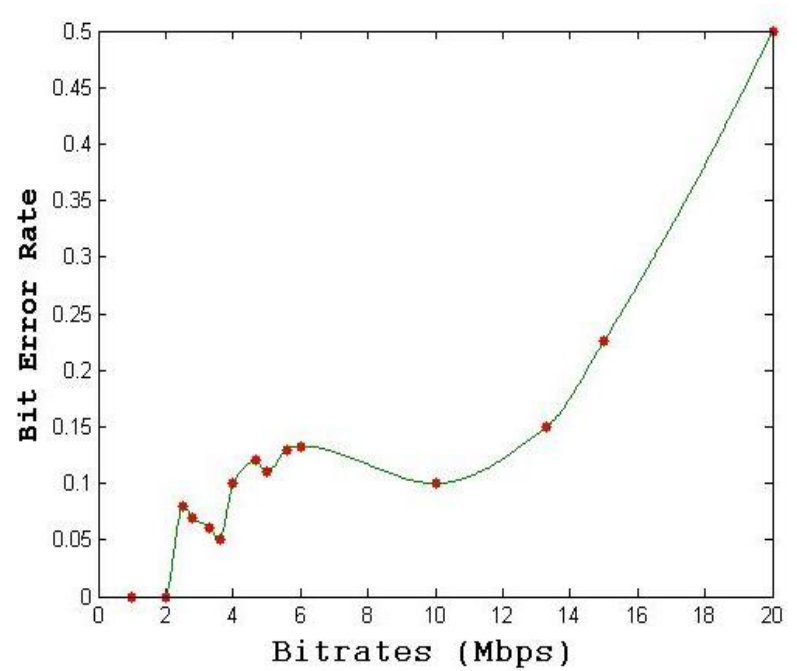

Fig. 22: Bit-error (BER) at different bit rates

\section{CONCLUSION}

RFID is a flexible wireless communication technology that is convenient, easy to use, and well suited for automatic operation. In the present work, MATLAB based RFID system is developed incorporating all the relevant ideas. Performance of the system is studied and tested with some test tag codes. Communication for code identification is investigated with the simulated model of our RFID System.

The effects of environmental noise on the system were also studied by incorporating different noise blocks in the model. A chaotic noise generator was designed and incorporated in the simulated model in order to realize actual environmental conditions. The performance of the simulated system is studied by measuring the BER. While at present bar code tags and bar code systems are much less expensive compared to RFID, it provides many benefits that bar code systems cannot. RFID technology makes immediate economic sense in areas where the cost of failure is great. Accordingly, for the foreseeable future, developing effective RFID applications will continue to be a stimulus and driver in RFID technology development.

\section{ACKNOWLEDGEMENT}

Tirthankar Datta and others thankfully acknowledge the untiring support of Prof. (Dr.) Subir Kumar Sarkar, HOD \& Professor, Department of Electronics \& Telecommunication Engineering, Jadavpur University and the technological infrastructure support obtained from Single Electron \& Spintronics Laboratory, Department of Electronics \& Telecommunication, Jadavpur University.

\section{REFERENCES}

[1] H. Stockman, "Communication by Means of Reflected Power", Proceedings of the IRE, pp. 1196-1204, October 1948.

[2] Cardullo et al., "Transponder apparatus and system", U.S. Patent 3,713,148, 1973.

[3] J. Landt, "Shrouds of Time: The history of RFID", AIM, Inc. 2001 .

[4] Bogdan C. Ionescu, "A Study of RFID Devices and Efficient Ways of Simulating RFID Systems with Maxwell Software", Technical Articles for E-M Products, Ansoft.

[5] S. Basat, K. Lim, I. Kim, M.M. Tentzeris, J. Laskar, "Design and Development of a Miniaturized Embedded UHF RFID Tag for Automotive Tire Applications", School of ECE, Georgia Institute of Technology, Atlanta, GA 30332-0250, USA

[6] Y. Tikhov, "Comments on "Antenna design for UHF RFID tags: A review and a practical application'," IEEE Trans. Antennas Propag., vol. 54, p. 1906, Jun. 2006.

[7] Richard Boss, "Library RFID technology", Library Technology Reports, Nov/Dec 2003.

[8] J. Halamka, A. Juels, A. Tubblefield, J. Westhues, "The security implications of VeriChip cloning", Journal of the American Medical Informatics Association, 2006. 
[9] G. P. Hancke, "Practical attacks on proximity identification systems", Proceedings of IEEE Symposium on Security and Privacy, pp. 328-333, 2006.

[10] I. Dinur, and K. Nissim, "Revealing information while preserving privacy", Proc. of the 22nd PODS Conf., pp. 202-210, 2003.

[11] Z. Min et al., "A RFID-based Material Tracking Information System", Proceedings of the IEEE International Conference on Automation and Logistics, Jinan, China, pp. 2922 - 2926, 2007.

[12] T. Datta et al., "RFID based Airport Logistics Management", 3rd Innovative Conference on Embedded Systems, Mobile Communication \& Computing (ICEMC2 2008), Infosys Mysore, Karnataka, India, pp. 228-232, 2008.

[13] M. T. Lockman, A. Selamat, "Multi-Agent Verification and Validation for RFID System Architecture", International Conference on Electronic Design, Penang, Malaysia, pp.1-5, 2008.

[14] C. C. Song et al., "Study and implementation of a networking information platform for RFID system", IEEE International Conference on Industrial Technology, ICIT 2008, pp. 1-6, 2008.

[15] S. M. Birari and S. Iyer," Mitigating the Reader Collision Problem in RFID Networks with Mobile Readers", 13th
IEEE International Conference on Networks, 2005 jointly held with the IEEE 7th Malaysia International Conference on Communication, 2005, vol. 1, pp. 6, 2005.

[16] T. Cheng; L. Jin, "Analysis and simulation of RFID anti-collision algorithms", The 9th International Conference on Advanced Communication Technology, pp. 697-701, 2007.

[17] Q. Xiao, C. Boulet, and T. Gibbons, "RFID Security Issues in Military Supply Chains", The Second International Conference on Availability, Reliability and Security, 2007, ARES 2007, pp. 599 - 605 , 2007.

[18] D. Barlas, "Wal-Mart's RFID mandate," 4 June 2003. [Online] available: http://www.line56.com/articles/default.asp?ArticleID $=4710$.

[19] T. Datta et al., "Realization \& Simulation of the Hardware for RFID system and its performance study", IET-UK International Conference on Information and Communication Technology in Electrical Sciences, organized by Dr. M. G. R. University, Chennai, Tamil Nadu, pp. 697 - 700, 2007.

[20] Y. Han, Q. Li, H. Min, "System modeling and simulation of RFID", Auto-ID Labs Research Workshop, Fudan University, Shanghai, 2004. 